\title{
A INDUSTRIALIZAÇÃO DA CONSTRUÇÃO COM TERRA ATRAVÉS DA IMPRESSÃO 3D
}

\section{INDUSTRIALIZATION OFEARTH CONSTRUCTION THROUGH 3D PRINTING}

\author{
Gladys I. K. Taparello, Arquiteta e Urbanista (UFSC) acadêmica do MBA em Gestão de Obras e \\ Projetos (UNISUL) \\ Palavras Chave \\ Impressão 3D; Construção com terra; Impressão 3D com terra.
}

\section{Key Words}

$3 D$ printing; Earth construction; $3 D$ printing with earth

\section{RESUMO}

A arquitetura tem incorporado novas estratégias e tecnologias para a materialidade do edifício e sustentabilidade no uso dos recursos. Dentre estas, a impressão 3D tem conseguido cada vez mais espaço nas indústrias em geral como um método inovador de fabricação, embora a tecnologia exista desde a década de 1980. Recentemente houve a introdução desta tecnologia no setor da construção civil, ainda em escala de testes. Entre os diversos materiais utilizados na impressão 3D de edificações destaca-se o solo, por ser um material barato, com grande disponibilidade, possibilidade de reciclagem total, com baixos impactos ambientais e ótimas propriedades de conforto térmico. Entretanto a construção com terra ainda é subaproveitada e vista com reservas, especialmente as técnicas mais artesanais. Assim este artigo propõe-se a explorar a prototipagem associado ao uso do solo de forma a aliar o baixo impacto ambiental e a racionalidade no uso do recurso com a diversidade da forma que a prototipagem pode conferir à arquitetura. Para cumprir com os objetivos revisou-se os principais conceitos da fabricação digital, as potencialidades do uso da terra como material de construção e por fim, o emprego deste material na prototipagem.

\section{ABSTRACT}

Architecture has incorporated new strategies and technologies for materializing buildings and sustainability of resources. Among those, 3D printing is achieving greater space in general industries as a innovative method of manufacturing, even though the technology had been developed in the 1980 decade. Recently this technology has been introduced into the civil construction still in experimental phase. About the many materials used in 3D printing of buildings earth is highlighted by its characteristics of low cost, great availability, possibility of full recycling, low environment impacts e great properties of insulation. However earth construction is still underrated, specially the handcrafted techniques. Therefore this article intend to exploit prototyping associated to the use of earth as a way of joining the low environment impacts and rationality of use of resources to the diversity of form that prototyping can give to architecture. To achieve the objectives, the main concepts of digital fabrication has been reviewed, as well as the potentialities of earth as a building material and use of this material in prototyping. 


\section{INTRODUÇÃO}

Este artigo propõe-se a explorar a prototipagem associado ao uso do solo de forma a aliar o baixo impacto ambiental e a racionalidade no uso do recurso com a diversidade da forma que a prototipagem pode conferir à arquitetura.

O objetivo geral é apresentar uma breve descrição das principais pesquisas relativas à fabricação digital na arquitetura. Os objetivos específicos são explicar sucintamente as aplicações da prototipagem na construção civil, apresentar as tecnologias atualmente em desenvolvimento, assim como os materiais com grande potencial de aplicação na impressão 3D na arquitetura.

Para cumprir com os objetivos revisou-se os principais conceitos da fabricação digital, as potencialidades do uso da terra como material de construção e por fim, o emprego deste material na prototipagem.

\section{IMPRESSÃO 3D}

A impressão 3D é o nome mais comum para designar produtos feitos através do sistema de prototipagem rápida, que é a fabricação de objetos físicos diretamente com o uso de informações geradas através de CAD (computer assisted design) (GORNI, 2003). Também pode ser chamado de Fabricação Aditiva por causa do seu método de fabricação que é basicamente a sobreposição de camadas de materiais para a geração do objeto, diferente dos modelos de produção por subtração ou por injeção de moldes, que já são mais conhecidos (LOPES, 2016).

Este método de fabricação teve origem nos anos 1980, com o processo de Estereolitografia patenteado pela empresa 3D Systems (LOPES, 2016). Além deste processo, que envolve a solidificação de polímeros líquidos fotossensíveis expostos à radiação ultravioleta, ainda há processos que se baseiam na sinterização de materiais pulverulentos através de aplicação de raios lasers (Sinterização Seletiva a Laser) ou de jatos de tinta (Impressão por Jato de Tinta), a extrusão de filamentos de resina termoplástica aquecida (Modelagem por Deposição de Material Fundido), a fusão de pó metálico através de lasers de alta potência (Conformação Próxima ao Formato Final via Laser), entre outros (GORNI, 2003).

Dependendo do processo de impressão 3D, há variadas matérias-primas a utilizar, como cera, poliestireno, nylon, vidro, cerâmica, terra, aço inoxidável, titânio, alumínio, plásticos de engenharia como ABS e PLA (os mais comuns para impressões 3D feitas em casa), e até mesmo matérias-primas comestíveis, como chocolate e queijo (TAKAGAKI, 2012). Dolhan (2013) e Takagaki (2012) listam algumas das indústrias que tem utilizado a impressão 3D em alguma etapa de produção: automobilística, desenho industrial, medicina, arqueologia, sistema de informações geográficas, aeroespacial, construção civil, entre outras.

O processo de prototipagem rápida se diferencia dos demais processos por sua facilidade de automação, o que reduz a participação do operador durante a produção (VOLPATO, 2007). As principais etapas deste sistema foram resumidas por Gorni (2003), como apresentado a seguir:

1. Criação de um modelo CAD da peça que está sendo projetada;

2. Conversão do arquivo CAD em formato $S T L$, próprio para estereolitografia;

3. Fatiamento do arquivo STL em finas camadas transversais;

4. Construção física do modelo, empilhando-se uma camada sobre a outra;

5. Limpeza e acabamento do protótipo. (GORNI, 2003)

No processo de desenvolvimento de produtos, especialmente na arquitetura, a elaboração de maquetes e modelos volumétricos durante a elaboração do projeto é de suma importância para que sejam lançadas as primeiras ideias de criação, assim como permitir análises baseadas em simulações de desempenho. Entretanto, o tempo consumido para a fabricação das maquetes, bem como o gasto com materiais de representação, que nem sempre possuem as mesmas propriedades dos materiais utilizados na construção final, se constituem como impedimentos para que a prática de modelagem física seja menos difundida.

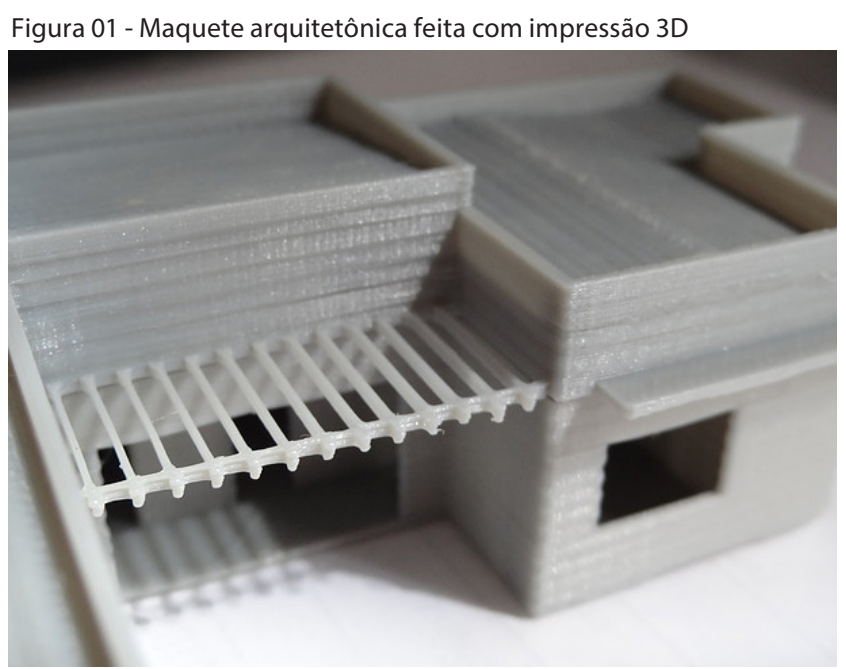

IMPRIMA1, 2016

Considerando este aspecto, de acordo com Volpato (2007), "a prototipagem rápida permite igualmente detectar erros de concepção numa fase inicial do processo de desenvolvimento, reduzindo deste modo os riscos associados ao lançamento de um novo produto", com a 
vantagem da diminuição do tempo de fabricação do modelo de estudo, assim como a possibilidade de se empregar materiais mais próximos aos que serão consumidos na fabricação final do produto.

Deste modo, a primeira aproximação da impressão 3D com a arquitetura é através da elaboração de maquetes de estudo, geralmente em pequenas escalas devido à restrição de tamanho das impressoras comuns atuais.

\section{CONSTRUÇÃO COM TERRA}

A construção com terra existe desde o período Neolítico e sobreviveu até os tempos atuais (GIANNAKOPOULOS, 2016). Estima-se que ao menos um terço da população mundial atual habite em construções feitas com terra (SANTOS, 2015). Apesar da descrença de que a construção com terra vem sendo alvo desde a descoberta de novos materiais construtivos, como aço e concreto, percebe-se que principalmente nas últimas décadas houve um aumento de interesse por técnicas construtivas que tem como base a terra crua (SANTOS, 2015). Isso porque, com o aumento de problemas como aquecimento global, escassez de matérias-primas não-renováveis, déficit habitacional, entre outros, começou a se buscar alternativas mais eficientes e com menos impacto ambiental. A terra surge então como uma solução muito apropriada, por suas características de resistência mecânica, regulação térmica, abundância de disponibilidade, baixas emissões de gases estufas, baixo custo, possibilidade de se reciclar totalmente as estruturas, entre muitas outras (GIANNAKOPOULOS, 2016).

Embora a terra como material seja excelente, os métodos construtivos que a empregam são, em sua maioria, técnicas rudimentares, com ares artesanais, e que muitas vezes possuem um acabamento com características grosseiras que não atraem esteticamente a população em geral. Por falta de conhecimento, estas técnicas são condenadas como inferiores a técnicas mais difundidas, como alvenaria de tijolo cerâmico e estrutura de concreto armado. Em consequência, pouco interesse se tem em desenvolver as técnicas tidas como rudimentares para uma situação de maior industrialização, que poderia diminuir o aspecto precário dos acabamentos e assim aumentar o apelo estético dessas técnicas, tornando-as mais fáceis de serem incorporadas ao público em geral.

Outro motivo de preconceito para com as técnicas com terra é o inseto barbeiro, transmissor do protozoário que causa a doença de Chagas. Mas como afirma o engenheiro civil Obede Borges Faria, "se mal feitas e mal conservadas, tanto uma casa de barro quanto uma de concreto deixam frestas onde o bicho pode se instalar. Não é um problema intrínseco do barro. É mais uma questão de acabamento e higiene" (ADOBE, 2013). Ademais, usando-se cal na pintura ou no reboco, como é normalmente aplicado nas construções com terra, previne a infestação com o inseto.

A flexibilidade da terra em relação ao modo de construir pode ser constatada ao se listar as técnicas que usam a terra como matéria-prima. Nito (2015) apresenta um diagrama elaborado pelo grupo CRATerre com os diferentes sistemas construtivos baseados em terra:

O grupo A de que trata a figura 2, reporta-se a sistemas monolíticos: 1) terra escavada; 2) terra plástica; 3) terra empilhada; 4) terra modelada; 5) terra compactada. O grupo B inclui os sistemas em alvenaria: 6) blocos apiloados; 7) blocos prensados; 8) blocos cortados; 9) torrões de terra; 10) terra extrudida; 11) adobe mecânico; 12) adobe manual; 13) adobe moldado. Por fim, integram o grupo C de sistemas mistos: 14) terra de recobrimento; 15) terra sobre engradado; 16) terra palha; 17) terra de enchimento; 18) terra de cobertura.

Figura 02 - Diagrama de sistemas construtivos em terra

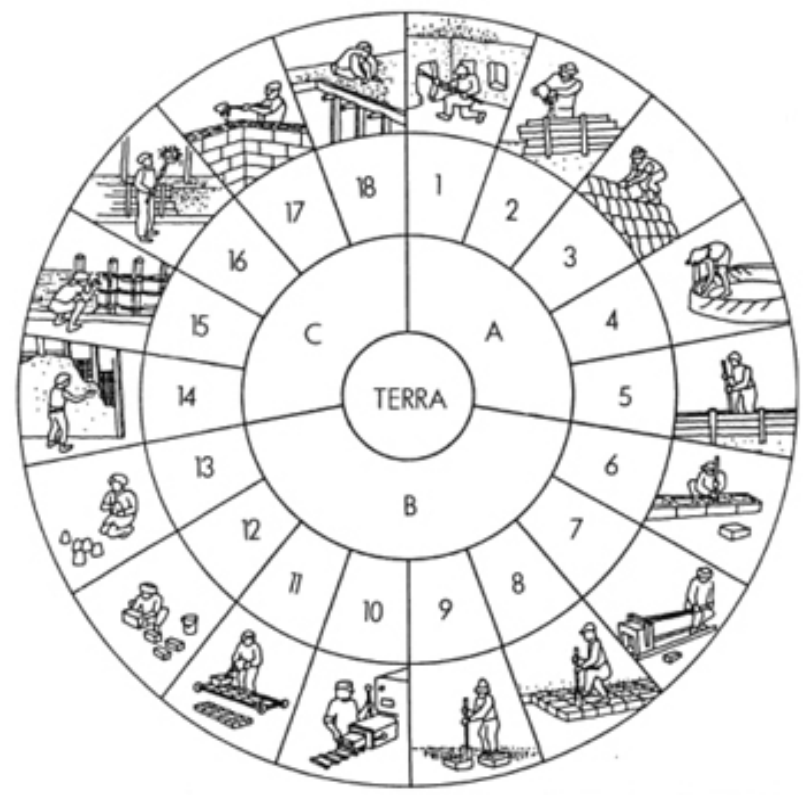

Fonte: NITO, 2015

Atualmente há uma grande variedade de cursos voltados para a bioconstrução e que empregam técnicas com terra, capacitando as pessoas para que possam construir suas próprias casas. O Ministério do Meio Ambiente desenvolveu, em 2008, uma cartilha que contém várias técnicas de autoconstrução, incluindo construções com adobe, superadobe, solocimento, taipa de mão e de pilão.

Há quem prefira empregar técnicas mais industrializadas na construção com terra, e também há soluções neste 
campo, com tijolos de solocimento facilmente encontrados à venda, e ainda empresas especializadas em construção industrializada com taipa.

\section{IMPRESSÃO 3D NA CONSTRUÇÃO CIVIL}

Apesar do entusiasmo de outros setores, e até mesmo da população como um todo, a construção civil ainda é reticente no uso da impressão 3D como forma de manufatura. Quer seja pelo conservadorismo do setor, quer seja pela necessidade de máquinas de maior porte do que as necessárias para as outras indústrias, o uso de impressoras 3D como sistema construtivo ainda é incipiente, uma vez que há poucos estudos e bibliografia disponível sobre o assunto (LOPES, 2016).

Ainda que hajam contratempos, algumas empresas, universidades e start ups têm se dedicado ao desenvolvimento da construção com impressão 3D. A chinesa Winsun ganhou destaque na mídia, em 2014, ao construir dez casas de $200 \mathrm{~m}^{2}$ em menos de $24 \mathrm{~h}$, usando como material construtivo concreto com agregados recicláveis. Para desenvolver o processo construtivo foram investidos cerca de US\$3,2 milhões em doze anos (SANTOS, 2014).

A University of Southern California desenvolve desde 2004 um projeto chamado Contour Crafting, desenvolvido pelo professor Behrokh Khoshnevis, inicialmente produzindo paredes de concreto autoportante para a construção de habitações de interesse social e de emergência pós-catástrofes a baixo custo e em menor tempo. Hoje em dia o grupo estuda junto com a Cal-Earth, uma organização da universidade voltada ao estudo das construções com terra, a construção de edificações com adobe através da impressão 3D.

Figura 03 - Casa construída pela Winsun

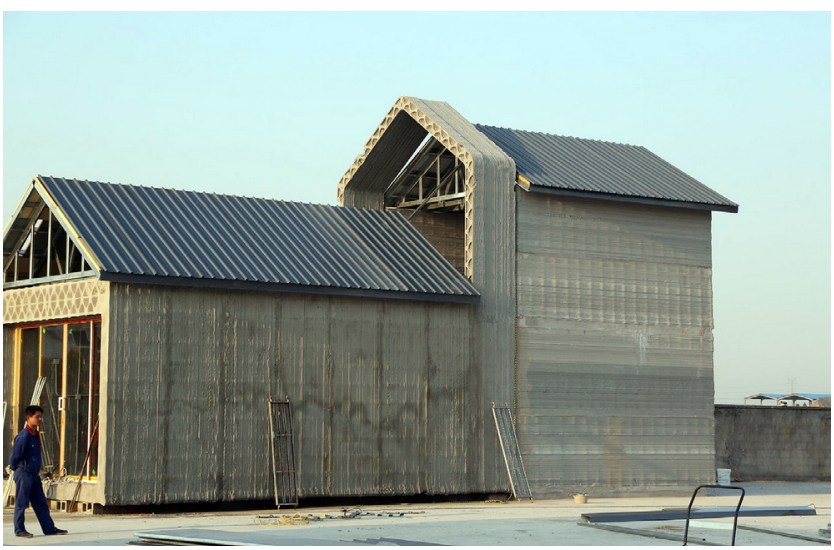

SANTOS, 2014

O escritório Gramazio Kohler Research, dos arquitetos Fabio Gramazio e Matthias Kohler, vem se dedicando, desde o início dos anos 2000, ao desenvolvimento de robôs construtores. Um dos projetos iniciais, de 2007, chamado
R-O-B, é uma máquina automatizada que produz tijolos no local da obra, e com a ajuda de um braço mecânico posiciona os tijolos nas posições predeterminadas.

Figura 04 - Esquema do processo construtivo de Contour Crafting

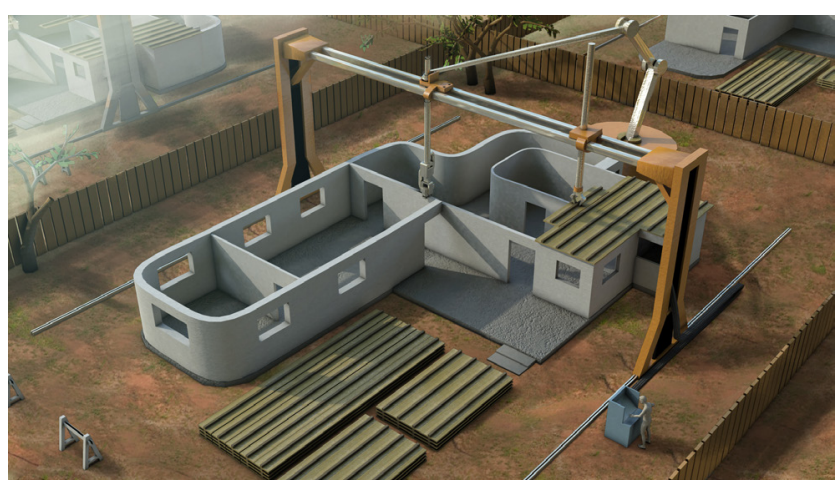

Fonte: CONTOUR, 2016

O IAAC (Institute for Advanced Architecture of Catalonia) em Barcelona, Espanha, tem entre seus projetos de pesquisa o desenvolvimento da impressão 3D em larga escala na construção civil. O de maior destaque é o projeto Pylos, que se baseia na utilização de um braço robótico para a impressão de edificações com terra. Atualmente o projeto se encontra em fase de testes de protótipos com estruturas em escala 1:1.A italiana WASP (World's Advanced Saving Project) é uma empresa voltada a ajuda humanitária, que propõe a construção de casas em áreas remotas, para populações carentes e vítimas de catástrofes naturais, através da impressão 3D com solo e fibras naturais. A máquina de impressão da empresa é uma das maiores disponíveis, com 12 metros de altura.

Figura 05 - R-O-B, de Gramazio Kohler Research

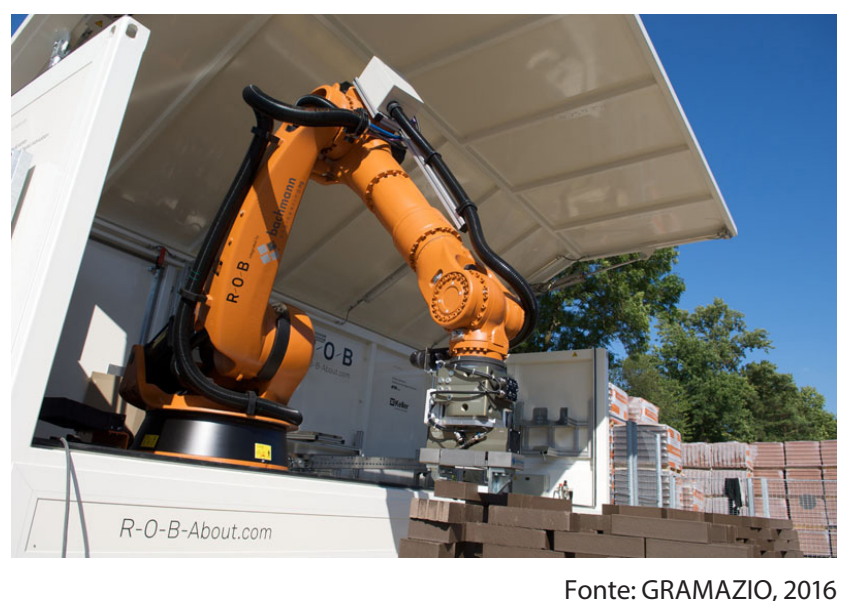

No Brasil, duas start ups se destacam na proposta de construção com impressão 3D, a InovaHouse 3D e a Urban 3D. Ambas tem como objetivo a construção de moradias de baixo custo e em menor tempo do que o processo padrão, utilizando materiais sustentáveis e resíduos 
reciclados. A Urban 3D trabalha em parceria com profissionais internacionais das áreas de química, robótica e concreto, e prevê ainda para 2016 a construção de um prédio de 12 andares. Já a InovaHouse 3D prevê para 2020 a finalização de sua primeira casa impressa em Brasília.

A construção de edificações com impressão 3D se torna mais atraente em situações em que é necessária qualidade do produto e rapidez no processo, como em casos de catástrofes naturais e moradias de interesse social. Por utilizar somente os materiais que serão consumidos na impressão, este processo evita desperdícios e se torna mais barato do que o processo construtivo padrão.

Figura 07 - A impressora de $12 \mathrm{~m}$ da WASP

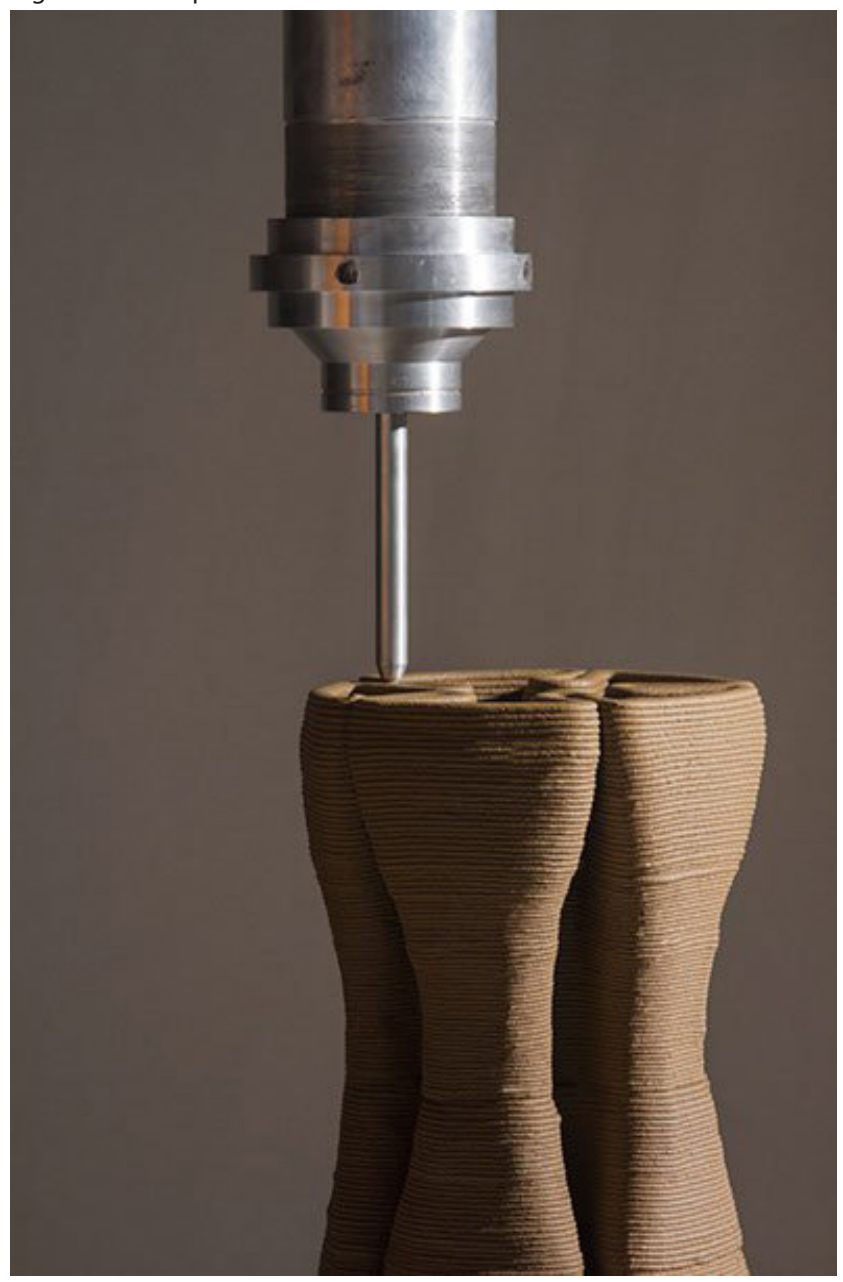

Fonte: WASP, 2016

O uso de cimento como material principal nas construções continua também na impressão 3D, embora nas impressões o uso de agregados reciclados seja maior do que nas construções de alvenaria e concreto moldado no local, que são mais comuns. A substituição do cimento, um produto com um alto impacto ambiental e grande consumo de energia no seu processo produtivo, por um produto de menor impacto como a terra pode diminuir ainda mais o custo da construção com impressão 3D.
O início da industrialização dos processos construtivos com terra abre as portas para o desenvolvimento de tecnologias cada vez mais avançadas, e aqui se inclui a impressão 3D com terra. Ao se minimizar o esforço humano na construção civil e aumentar a capacitação profissional dos trabaIhadores, também se promove a melhoria das condições de vida destes mesmos trabalhadores, ao terem maior acesso a informações e desenvolverem o pensamento crítico.

\section{CONSIDERAÇÕES FINAIS}

A prototipagem representa um novo mundo a ser explorado na construção civil, tanto para fabricação da habitação como de seus componentes. Os processos vão desde a utilização de resinas e polímeros, até o emprego de materiais tradicionais (como blocos e tijolos colados) ou argamassas projetadas em espaços preconcebidos. Se aliada ao uso de materiais menos impactantes, a fabricação digital representa um importante caminho para melhoria da qualidade das edificações e saneamento do déficit habitacional mundial.

\section{REFERÊNCIAS}

GORNI, Antonio Augusto. Introdução à prototipagem rápida e seus processos. 2003. Disponível em: <http:// www.gorni.eng.br/protrap.html>. Acesso em: 13 jun. 2016.

LOPES, Gonçalo Teixeira Ferreira. Exploração das possibilidades da impressão 3D na construção civil. 2016. 92 f. Dissertação (Mestrado) - Curso de Faculdade de Engenharia, Engenharia Civil, Universidade do Porto, Porto, Portugal, 2016. Disponível em: <https:// sigarra.up.pt/feup/pt/pub_geral.show_file?pi_gdoc_ id=729932>. Acesso em: 13 jun. 2016.

TAKAGAKI, Luiz Koiti. Tecnologia de Impressão 3D. Inovação Tecnológica, São Paulo, v. 2, n. 2, p.28-40, dez. 2012. Semestral. Disponível em: <http://antigo.faculdadeflamingo.com.br/ojs/index.php/rit/article/viewFile/54/71>. Acesso em: 13 jun. 2016.

DOLHAN, Valentin. 3D Printing in Architecture: A current state of the industry with past and future perspective. 2013. 105 f. TCC (Graduação) - Curso de Architectural Technology \& Construction Management, Via University College, Horsens, Dinamarca, 2013. Disponível em: $<$ http://www.academia.edu/10243998/3D_Printing_in Architecture_-_A_current_state_of_the_industry_with past_and_future_perspective>. Acesso em: 02 jul. 2016. 
VOLPATO, Neri. Prototipagem rápida: Tecnologias e aplicações. São Paulo: Blücher, 2007.

IMPRIMA1: Impressão 3d e prototipagem. Impressão 3d e prototipagem. Disponível em: <http://www.imprima1.com/>. Acesso em: 2 jul. 2016.

SANTOS, Clarissa Armando dos. Construção com terra no Brasil: panorama, normatização e prototipagem com terra ensacada. 2015. 290 f. Dissertação (Mestrado) - Curso de Programa de Pós-graduação em Arquitetura e Urbanismo, Universidade Federal de Santa Catarina, Florianópolis, 2015. Disponível em: <https://repositorio.ufsc. br/handle/123456789/159436>. Acesso em: 24 jun. 2016.

$A D O B E$, matéria-prima tão antiga, pode ser alternativa para o futuro. jun. 2013. Disponível em: <http:// casa.abril.com.br/materia/adobe-materia-prima-tao-antiga-pode-ser-alternativa-para-o-futuro>. Acesso em: 24 jun. 2016.

NITO, Mariana Kimie da Silva. Sistemas construtivos em terra crua: panorama da América Latina nos últimos $\mathbf{3 0}$ anos e suas referências técnicas históricas. Cadernos de Pesquisa da Escola da Cidade, São Paulo, SP, n. 1, p.11-19, 2015. Disponível em: <http://www.escoladacidade.org/wp/wp-content/uploads/160323_publicacao_pesquisa_completo.pdf>. Acesso em: 24 jun. 2016.

BRASIL. Curso de Bioconstrução. Brasília: Ministério do Meio Ambiente, 2008. 64 p. Texto elaborado por: Cecília Prompt. Disponível em: <http://www.mma.gov. br/estruturas/sedr_proecotur/_publicacao/140_publicacao15012009110921.pdf>. Acesso em: 24 jun. 2016.

TAIPAL. Disponível em: <http://www.taipal.com.br/>. Acesso em: 24 jun. 2016.

SANTOS, Altair. Impressora 3D constrói casas com concreto reciclado. Nov. 2014. Disponível em: <http:// www.cimentoitambe.com.br/impressora-3d-constroi-casas/>. Acesso em: 15 jun. 2016.

CONTOUR Crafting: How does it work?. Disponível em: <http://www.contourcrafting.org/how-does-it-work/>. Acesso em: 15 jun. 2016.

GRAMAZIO, Fabio; KOHLER, Matthias. R-O-B: Mobile Fabrication Unit. 2007. Disponível em: <http:// gramaziokohler.arch.ethz.ch/web/e/forschung/135. html>. Acesso em: 19 ago. 2016.

GIANNAKOPOULOS, Sofoklis. Pylos: 3D Printing with soil and natural materials. Disponível em: <http:// pylos.iaac.net/>. Acesso em: 24 jun. 2016.

WASP (World's Advanced Saving Project). Disponível em:<http://www.wasproject.it/w/en/>. Acesso em: 02 jul. 2016.

PINHEIRO, Luisa. CASA IMPRESSA EM 3D? SIM, JÁ TEM. Disponível em: <http://infograficos.estadao.com. br/e/focas/movimento-maker/casa-impressa-em-3d-sim-ja-tem.php\#>. Acesso em: 02 jul. 2016.

URBAN 3D. Disponível em: <http://www.urban3d. co/>. Acesso em: 02 jul. 2016.

BECK, Bibiana. EMPATIA, INTELIGÊNCIA E TECNOLOGIA A FAVOR DO IMPACTO SOCIAL: CONHEÇA ANIELLE GUEDES. 2015. Disponível em: <http://socialgoodbrasil.org.br/2015/empatia-inteligencia-e-tecnologia-favor-impacto-social-conheca-anielle-guedes>. Acesso em: 02 jul. 2016.

INOVAHOUSE 3D. Disponível em: <http://inovahouse3d.com.br/>. Acesso em: 02 jul. 2016.

MORE: Mecanismo online para referências, versão 2.0. Florianópolis: UFSC Rexlab, 2013. 